\title{
DEVELOPMENT OF A SYSTEM TO STUDY THE IMPACT OF HEADLIGHT GLARE IN A DRIVING SIMULATOR
}

\author{
Matthew Fullerton ${ }^{1,2,3}$ and Eli Peli ${ }^{1}$ \\ ${ }^{1}$ Schepens Eye Research Institute, Harvard Medical School \\ Boston MA, USA \\ 2 Department of Electronics \\ University of York, UK \\ ${ }^{3}$ Department of Traffic Engineering \\ Technische Universität München, Germany \\ Email: eli.peli@schepens.harvard.edu
}

\begin{abstract}
Summary: We report on the development of a realistic dynamic simulation of oncoming headlamp glare in a driving simulator. To our knowledge, no such simulation had been attempted or achieved previously. The simulation is based on superposition of a bright LED display through a beam splitter on the simulator screen and synchronizing the illuminated LED position to the image of the simulated oncoming car. LED brightness is adjusted to result in the level of light that such headlights would cause in the driver's eyes, enabling the testing of glare effect on drivers of different ages and impact (reduction or increase) of various vision devices.
\end{abstract}

\section{INTRODUCTION}

Anyone who drives at night, especially on quieter roads, is familiar with oncoming blinding headlights. The blinding effect may be almost total if the lights have not been switched from high beam, but even on low beam there is substantial discomfort and reduced visibility. We have designed and implemented an accurate simulation of these effects in a safe and repeatable way. To our knowledge the behavioral effects of headlight glare have never directly been examined, and no measure of eye and head movement during driving glare was found in the literature. Further, behavioral studies that are summarized below have used inaccurate glare simulations.

\section{What is Glare?}

Glare can be described as the visual effect of any relatively bright light source in the visual field that causes some form of disturbance to visual activities. Glaring sources can reduce our ability to see other objects ('disability' glare), or merely cause annoyance ('discomfort' glare) (Vos, 2003). Glare from oncoming car headlamps has been the source examined most closely (because of the safety implications) (Mace et al., 2001). Light scatter inside and out of the eye (i.e., in the windshield) causes a veiling glare which reduces retinal contrast across the visual scene and thus reduces visibility. The pupillary response to the bright source might be the main cause for the discomfort associated with glaring lights (Hopkinson \& Petherbridge, 1956). Due to the reduction in visibility of on-road objects, disability glare has clear safety implications, but discomfort glare may cause avoidance behaviors that may also reduce overall visibility and reduce safety, simply because the driver has shifted his view away from important objects (Theeuwes et al., 2002). 


\section{Quantifying the Behavioral Impact}

People are affected in different ways by glare. Changes in the ocular media, brought about by age (Vos, 2003), eye surgery (Ginsburg \& Subramaniam, 2007), cataract (Sjostrand, et al., 1987) or the use of contact lenses (Applegate \& Wolf, 1987), intraocular lenses (Nadler et al., 1984; Schmitz et al., 2000) and spectacles (Vos \& Padmos, 1983), can all worsen the 'veiling luminance' upon the retina responsible for disability glare. Effects of variations in design are of interest to manufacturers of ophthalmic devices, as they are required to satisfy FDA safety concerns and also want to verify that their products are not worsening the night-driving situation. Addressing these issues requires methods to measure the effect of glare on a person, with and without such ophthalmic devices. Approaches include the measurement of intraocular light scatter that is responsible for disability glare and asking drivers to subjectively rate their glare problems, or the annoyance of a particular glare source (Aslam et al., 2007). Acuity or contrast sensitivity tests can also be performed with glare sources of different magnitude and different angles from the test target present (Aslam et al., 2007).

Attempts have been made to correlate the results of such clinical tests with driving performance in, and glare tolerance during, various driving tasks (Featherstone et al., 1999; Ginsburg \& Subramaniam, 2007; Pulling et al., 1980; Theeuwes et al., 2002). Measuring contrast sensitivity (Kaupp et al., 2005; Wood \& Owens, 2005) or mesopic visual acuity (Wood \& Owens, 2005) correlates somewhat with performance, but is not a reliable predictor (Featherstone et al., 1999). Theeuwes et al. (2002) made the interesting discovery that drivers' impression of a glare source does not relate well to their on-road performance. Pulling et al. (1980) found a large variation between clinical (Wolf tester) glare thresholds and on-the-road glare thresholds, measured by increasing the level of light deflected into a driver's eyes while in a driving simulator. The Mesotest II (Oculus Optikgeräte, $\mathrm{GmbH}$, Germany), a 'clinical' device simulating night driving lighting conditions, only showed limited correlation with patients' own perception of their disability at night (van Rijn et al., 2002).

The lack of a solid relationship between clinical tests and driving behavior prevents clinicians from making judgments of an individual's ability to drive in the presence of glare because these tests cannot directly measure the effect on functional performance. One problem in the simple tests is that the glare experience is not similar to what one experiences when driving at night. There are variations in source angle, intensity and spread that are not accurately matched to car headlights. Furthermore, in more elaborate real and simulated driving tasks, the glare sources used have been somewhat realistic but static (e.g., Featherstone et al., 1999; Pulling et al., 1980; Theeuwes et al., 2002; Wood \& Owens, 2005). The current 'standard' test that has been used in a number of FDA studies, the 'Night Driving Simulator' of Vision Sciences Research Corp. (VSRC, San Ramon, CA), does not accurately simulate glare in terms of the light intensity (headlights are only shown as part of the film capture). Even if a realistic glare source were to be used in a clinical test, it would not give us a precise picture of the functional implications of the glare. Such clinical tests do not allow an investigator to study any meaningful change in driving ability, such as reaction times to realistic objects in view (pedestrians and animals), steering stability, and distraction behaviors (head and eye movements away from the direction of travel). Even the VSRC system does not provide driving performance measures, such as gaze information and steering stability, only reaction times. As the subject does not have control of 
the simulated vehicle in this test, the validity of those reaction times can also be called into question. The goal of this project was to design and implement a system that will safely present highly realistic headlight glare, while fully measuring driving performance. Here, we detail our solution, creating an accurate glare simulation around a FAAC PP1000 driving simulator (FAAC, Ann Arbor, MI).

Table 1. A tabulation of the necessary light levels for longitudinal road separations of $1000 \mathrm{~m}-5 \mathrm{~m}$. Using trigonometry, the lamp eccentricity is computed. The incident illuminance is calculated by looking up the incident portion of the beam intensity at this angle (Schoettle et al., 2001) (data taken from Table $8, \mathbf{5 0}^{\text {th }}$ percentile and interpolated using MATLAB (Mathworks, Natick, MA)). Also given is the 'Veiling Luminance' on the retina due to light scatter in the eye, for a 30 year old person with blue eyes, using the CIE General Equation for Glare (Johannes J. Vos, 2003). Using this known illuminance at the eye, we calculate the necessary beam intensity at the simulator screen distance of $0.8 \mathrm{~m}$ to create that same illuminance. By imagining we have a luminance meter with an angle equal to the headlamp extent, we infer the luminance needed at the display to create the illuminance incident at the sensor. The calculations are based on road and car dimensions (distances from eye level to headlamp, headlamp size etc.) from the driving simulator scenario.

\begin{tabular}{|c|c|c|c|c|c|c|c|}
\hline $\begin{array}{l}\text { Road } \\
\text { Distance } \\
\text { (m) }\end{array}$ & $\begin{array}{c}\text { Eccentricity } \\
\left({ }^{\circ}\right)\end{array}$ & $\begin{array}{l}\text { Intensity at } \\
\text { this Angle } \\
\text { (cd) }\end{array}$ & $\begin{array}{l}\text { Illuminance } \\
\qquad(\mathbf{l x})\end{array}$ & $\begin{array}{c}\text { Veiling } \\
\text { Luminance } \\
\left(\mathbf{c d} / \mathbf{m}^{\wedge} 2\right)\end{array}$ & $\begin{array}{l}\text { Reqd. Intensity } \\
\text { (a) } 0.80 \mathrm{~m} \text { (cd) }\end{array}$ & $\begin{array}{c}\text { Sample Angle } \\
\left({ }^{\circ}\right)\end{array}$ & $\begin{array}{l}\text { Reqd. Display } \\
\text { Luminance } \\
\left(c d / \mathbf{m}^{\wedge} 2\right)\end{array}$ \\
\hline 1000 & 0.21 & 2191 & 0.00 & 2.67 & 0.00 & 0.02 & 15968 \\
\hline 500 & 0.42 & 1692 & 0.01 & 1.13 & 0.00 & 0.05 & 12331 \\
\hline 300 & 0.70 & 1474 & 0.02 & 0.66 & 0.01 & 0.08 & 10742 \\
\hline 180 & 1.16 & 1302 & 0.04 & 0.41 & 0.03 & 0.13 & 9491 \\
\hline 120 & 1.74 & 967 & 0.07 & 0.24 & 0.04 & 0.20 & 7051 \\
\hline 90 & 2.32 & 837 & 0.10 & 0.18 & 0.07 & 0.27 & 6112 \\
\hline 70 & 2.98 & 651 & 0.13 & 0.13 & 0.08 & 0.34 & 4758 \\
\hline 40 & 5.21 & 415 & 0.26 & 0.07 & 0.16 & 0.59 & 3047 \\
\hline 20 & 10.33 & 237 & 0.57 & 0.03 & 0.37 & 1.16 & 1785 \\
\hline 15 & 13.66 & 137 & 0.58 & 0.02 & 0.37 & 1.51 & 1059 \\
\hline 10 & 20.02 & 84 & 0.74 & 0.01 & 0.47 & 2.12 & 687 \\
\hline 8 & 24.49 & 62 & 0.80 & 0.01 & 0.51 & 2.49 & 543 \\
\hline 5 & 36.09 & 37 & 0.96 & 0.00 & 0.61 & 3.15 & 404 \\
\hline
\end{tabular}

\section{METHOD}

Normally, our simulator depicts headlights as on-screen white pixels. Using data about headlamp intensity across the beam pattern (Schoettle et al., 2001), we have determined the necessary luminance (for a display) and luminous intensity (for a beam of light) for a series of points along the road (Table 1). One can see that by the time the oncoming headlamps move off our simulator's central monitor (subtending $45^{\circ}$ ) they would no longer be a significant source of veiling glare $\left(0.01 \mathrm{~cd} / \mathrm{m}_{2}\right)$, implying that the glare simulation is needed only for the central monitor. Up to that point, around $10 \mathrm{~m}$ away, the necessary luminances range from $687 \mathrm{~cd} / \mathrm{m} 2$ to around $10000 \mathrm{~cd} / \mathrm{m} 2$ (assuming we only consider distances $300 \mathrm{~m}$ and below). A typical CRT has a luminance of 50 to $100 \mathrm{~cd} / \mathrm{m} 2$, modern LCD displays around 200 to $600 \mathrm{~cd} / \mathrm{m} 2$, with only experimental displays with individually controlled LED backlights (for high dynamic range images) capable of $10000 \mathrm{~cd} / \mathrm{m} 2$ (Seetzen et al., 2004). Thus, current display technology cannot yet achieve the necessary luminance to create realistic headlight glare directly on the screen. 
This has led us to our design that incorporates a separate glare source (a secondary display) of sufficient brightness superimposed over the central simulator monitor using a 50/50 optical beam splitter (Fig. 1), aligned and synchronized with the dynamic simulation of the oncoming car accounting for the varying driver position on the road. This form of optical setup also avoids any in-display interactions between the light sources; all interactions are limited to the driver's eyes.

Figure 1. Optical Setup of System. Using a 50/50 beam splitter at $45^{\circ}, 50 \%$ of the glare source is reflected and $50 \%$ of the screen luminance passes through the glass. The remaining components of both sources are directed away from the viewer. While this reduces the display simulator brightness by $50 \%$, such a reduction is of little concern when simulating night driving. In addition, the just noticeable difference in brightness is around a factor of 2 (Gilchrist, 2006), so the reduction in brightness (though easily detectable when compared to the side screens) would be barely noticeable on its own.We are also able to adjust the side screens such that their luminance matches that of the central screen.

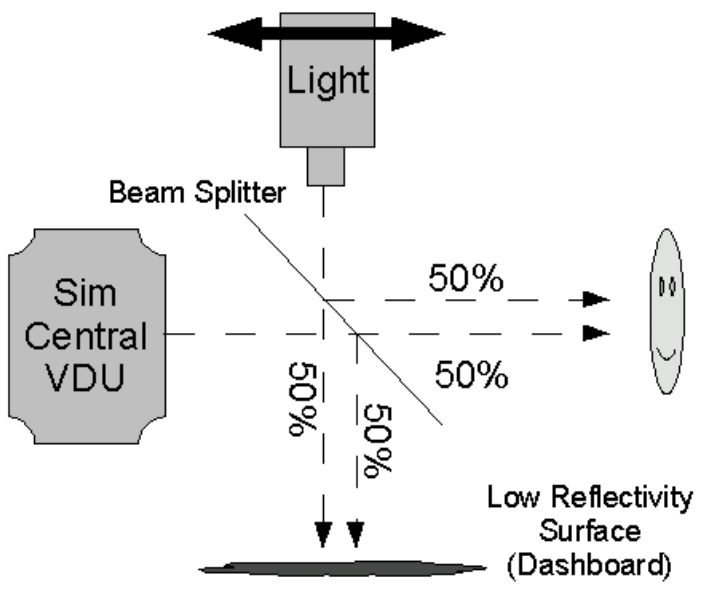

Synchronization. The necessary information to control this secondary display needs to be based on real-time information from the simulator computer. This was not only necessary to obtain the path of an oncoming car, but essential in order to continue the simulation if the driver steers away from his normal driven path (a possibility that we are interested in as a research outcome). From the live simulator network data stream, we obtain the distance along and the distance across the road separating the cars. This requires a number of geometrical operations to transform positions on a 2D map to distances relative to the driver's position. The position to place the headlamps on screen is simply the position of the angle they make in real life through the simulator screen (though in our software, we use a pinhole camera model to derive the same information). Sample results are given in Table 1.

Display. The current headlight simulation system uses a 'Peggy 2' LED display kit (http://www.evilmadscientist.com/article.php/peggy2). The display provides a 25 by 25 grid of $10 \mathrm{~mm} 2$ slots for standard 20mA LEDs, and is built around a programmable microcontroller. It also supports the Arduino programming system, a software layer/library that provides a straightforward programming interface. The display enables one row at a time, and an array of enabled columns will be lit in that row (hence there is a 25:1 reduction in maximum LED intensity when all rows are used by sequential access, since a row is only powered 1/25 of the time). Our software currently uses only the required rows (controlling position), in order to keep LEDs lit for as long as possible, and controls the frequency at which those LEDs are lit (in order to control intensity). Positions and levels of intensity are provided by custom Java software that performs the synchronization described above, and finds the correct intensity using the interpolated look-up table. Data is sent over a serial interface to the board. A calibration tool is included to infer the intensity the LEDs are actually providing given the measured illuminance at the driver's head position. 
LED Choice and Intensity Control. When one looks at the sizes of the simulated objects required (Table 1), it can be seen that rarely will more than one LED be used, due to the $10 \mathrm{~mm}$ spacing. The LEDs are mounted perpendicular to the board, and so are not all directed toward the driver. We are simulating something decreasing in distance, so the intensity of the source must increase, but the angle made with the LEDs is increasing, as the currently lit LED moves outward in the visual field, creating a fall-off in luminous intensity directed at the driver. This is to some extent an advantage in that it approximates the real-world situation of luminous intensity directed at the driver decreasing as the car gets closer to the oncoming vehicle due to the fall-off of the headlight beam itself (Table 1). In this way we create only a simulation of one portion of the headlight beam pattern, and not of the pattern itself, but it should naturally simulate at least qualitatively the correct behavioral response (head moves closer to LED axis, light increases, head moves further away, light decreases). The beam pattern of most LEDs can be described by the summation of Gaussian or cosine power functions (Moreno \& Sun, 2008). We therefore calculated the reduction in intensity at the angle the currently lit LED makes for a nominal head position in order to (prior to LED choice) ensure that the correct intensity can still be achieved for a given LED and (during simulation) choose the intensity level for the chosen LED. The LED chosen for the display was selected by first finding LEDs that had extremely high intensities. Generally this was only possible with narrow beam LEDs; which was appropriate as the angle of view should never exceed half the screen angle of $22.5^{\circ}$. We then fitted cosine power functions to the manufacturers' data. In addition to taking account of the angular fall-off, we sought to have an LED bright enough to account for light losses from utilizing all 25 LED rows sequentially (see Display, above), and the 50\% loss at the beam splitter. We found only two LEDs that would suffice, and of those two the Nichia NSPW500DS did so more comfortably. This LED was therefore selected for use in the display. As there is some evidence for newer headlamps with higher color temperatures causing more discomfort (Sivak et al., 2005), we obtained LEDs from the manufacturer that were presorted for highest intensity and lowest color temperature.

Head and Eye Tracking. In order to study head and eye movements, we have fitted our simulator with a Smart Eye ${ }^{\mathrm{TM}}$ combined head and eye tracking system (Smart Eye AB, Sweden). It uses two remote cameras to achieve gaze tracking with accuracies $<1.0^{\circ}$.

\section{OUTCOME}

Figure 2 below shows the system in action. The contrast between the screen and the headlamps is not fully appreciable in photographs due to the camera's limited dynamic range, and the experience is different for the driver than it is for the onlooker position shown here (due to the different angle made with the LEDs). Drivers testify to being unable to see the area around the car headlamps. 

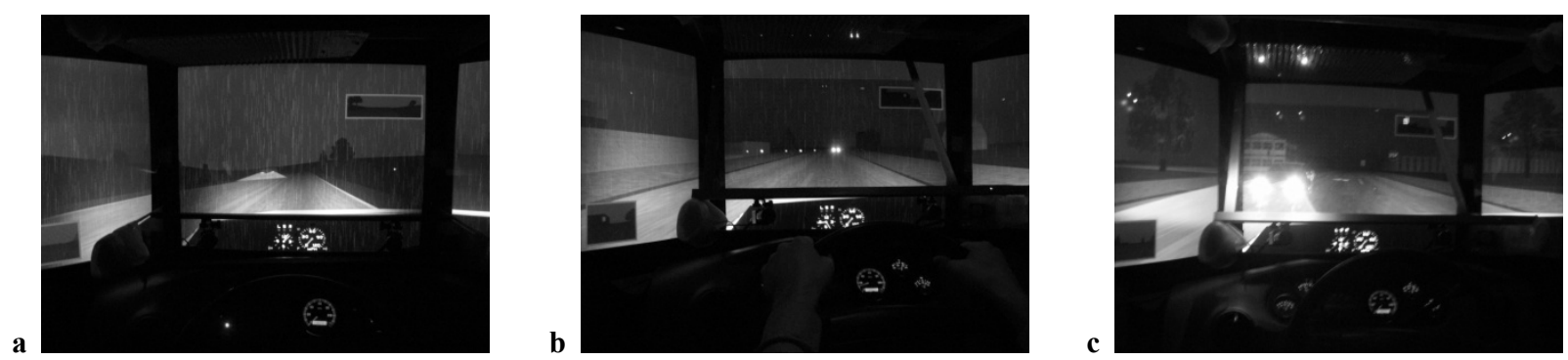

Figure 2. The night driving scene (a) without the glare system enabled, (b) simulating a distant vehicle, (c) simulating a close vehicle. The display and beam splitter can also be seen in (b) and (c).

\section{DISCUSSION}

The system described above has added a dramatic simulation of headlight glare to an existing driving simulator. One limitation of the system is the coarse spacing (10mm) between LEDs, which can make the motion of the headlights less smooth than we would like. This was deemed sufficient for a first prototype. Possibilities we are examining to resolve this include overlaying a second board in the spaces between LEDs, or making a smaller board with equal numbers of LEDs but in a smaller area. The approximation of increasing and decreasing headlight intensity fall-off as the driver deviates from the assumed position may prove to be insufficient. An additional possible use for the head tracking data would be to adjust the simulated portion of the beam pattern according the actual, rather than assumed head position instead of making this approximation.

\section{REFERENCES}

Applegate, R. A., \& Wolf, M. (1987). Disability glare increased by hydrogel lens wear. Am J Optom Physiol Opt, 64(5), 309-312.

Aslam, T. M., Haider, D., \& Murray, I. J. (2007). Principles of disability glare measurement: an ophthalmological perspective. Acta Ophthalmologica Scandinavica, 85(4), 354-360.

Featherstone, K. A., Bloomfield, J. R., Lang, A. J., Miller-Meeks, M. J., Woodworth, G., \& Steinert, R. F. (1999). Driving simulation study: bilateral array multifocal versus bilateral AMO monofocal intraocular lenses. Journal of Cataract \& Refractive Surgery, 25(9), 12541262.

Gilchrist, A. L. (2006). Seeing in Black and White. New York: Oxford University Press

Ginsburg, A. P., \& Subramaniam, S. V. (2007). Visual acuity, contrast sensitivity and night driving visibility in complaining LASIK subjects (Abstract). Investigative OphthalmologInvestigative Ophthalmology \& Visual Science, 48(E), 1575.

Hopkinson, R. G., \& Petherbridge, P. (1956). Sky Luminance Measurements. Journal of the Optical Society of America, 46(7), 555-556.

Kaupp, S. E., Schallhorn, S. C., Tanzer, D. J., van de Pol, C., Malady, S. E., Lombardo, K. M., et al. (2005). Prospective Comparison of Simulated Night Driving Performance After LASIK Treatment for High Myopia Using Four Different Excimer Laser Systems (Abstract) (Vol. 46, pp. 4344). 
Mace, D., Garvey, P., Porter, R., Schwab, R., \& Werner, A. (2001). Countermeasures for Reducing the Effects of Headlight Glare. Washington, D.C.: The AAA Foundation for Traffic Safety.

Moreno, I., \& Sun, C. C. (2008). Modeling the radiation pattern of LEDs. Opt Express, 16(3), 1808-1819.

Nadler, D. J., Jaffe, N. S., Clayman, H. M., Jaffe, M. S., \& Luscombe, S. M. (1984). Glare disability in eyes with intraocular lenses. Am J Ophthalmol, 97(1), 43-47.

Pulling, N. H., Wolf, E., Sturgis, S. P., Vaillancourt, D. R., \& Dolliver, J. J. (1980). Headlight glare resistance and driver age. Human Factors, 22(1), 103-112.

Schmitz, S., Dick, H. B., Krummenauer, F., Schwenn, O., \& Krist, R. (2000). Contrast sensitivity and glare disability by halogen light after monofocal and multifocal lens implantation. $\mathrm{Br} J$ Ophthalmol, 84(10), 1109-1112.

Schoettle, B., Sivak, M., Flannagan, M. J. (2001). High-beam and low-beam headlight patterns in the US and Europe at the turn of the millennium (Technical Report No. UMTRI-2001-19). Ann Arbor, MI: University of Michigan Transportation Research Institute.

Seetzen, H., Heidrich, W., Stuerzlinger, W., Ward, G., Whitehead, L., Trentacoste, M., et al. (2004). High dynamic range display systems. ACM Transactions on Graphics (TOG), 23(3), 760-768.

Sivak, M., Schoettle, B., Minoda, T., \& Flannagan, M. J. (2005). Blue content of LED headlamps and discomfort glare (Technical Report No. UMTRI-2005-2). Ann Arrbor, MI: University of Michigan Transportation Research Institute.

Sjostrand, J., Abrahamsson, M., \& Hard, A. L. (1987). Glare disability as a cause of deterioration of vision in cataract patients. Acta Ophthalmol Suppl, 182, 103-106.

Theeuwes, J., Alferdinck, J. W., \& Perel, M. (2002). Relation between glare and driving performance. Human Factors, 44(1), 95-107.

van Rijn, L. J., Wilhelm, H., Emesz, M., Kaper, R., Heine, S., Nitsch, S., et al. (2002). Relation between perceived driving disability and scores of vision screening tests. British Journal of Ophthalmology, 86(11), 1262-1264.

Vos, J. J. (2003). On the cause of disability glare and its dependence on glare angle, age and ocular pigmentation. Clinical and Experimental Ophthalmology, 86(6), 363-370.

Vos, J. J. (2003). Reflections on glare. Lighting Research and Technology, 35(2), 163-176.

Vos, J. J., \& Padmos, P. (1983). Stray light sensitivity and the critical object in relation to tunnel entrance lighting. DIE Transactions of the InternationalCommision on Illumination, 20th Session.

Wood, J. M., \& Owens, D. A. (2005). Standard measures of visual acuity do not predict drivers' recognition performance under day or night conditions. Optometry and Vision Science, 82(8), 698-705. 